\title{
Based on the topographic factors NDVI spatial distribution characteristics in Nanchong city, Sichuan province, China
}

\author{
Jun Zhang ${ }^{1, \text { a }}$, Chunrong Jia ${ }^{1, b}$, Quan Liu ${ }^{2, c}$, Zehong Zhang ${ }^{1, d}$ \\ ${ }^{1}$ College of Land and Resources, China West Normal University, Nanchong, Sichuan, China \\ ${ }^{2}$ Mianyang Normal University, Mianyang, Sichuan, China \\ azhang822@yeah.net, ${ }^{b}$ pyozyl@126.com, ${ }^{c}$ liuquan78@126.com, ${ }^{\mathrm{d}}$ zhangzehong2001@126.com
}

\begin{abstract}
Keywords: Nanchong city; NDVI; topographic factors
Abstract. Vegetation coverage is the main body of terrestrial ecosystem. Detecting the spatial distribution pattern of vegetation will benefit the regional ecological environment protection and soil erosion control. This study, taking the Nanchong city as the research area, MODIS NDVI (resolution $500 \mathrm{~m}$ ) date and GIS technology were used to obtain spatial distribution features of the different NDVI degree in the case of elevation, slope degree and slope direction via overlap of distribution maps of NDVI and topographic factors maps extracted from digital elevation model. The results showed that (1) the average NDVI value was 0.76 , which indicated that the ecological environment was better in Nanchong city. The high vegetation coverage distributed in the eastern region, while the middle vegetation coverage distributed in the western region. Low vegetation coverage distributed in the Jialing River valley, especially in urban area. (2)At the different elevation and slope degree, NDVI mean is rising. In which the largest vegetation coverage area of high, medium and low appeared at 300-500 $\mathrm{m}$ in different altitude. And in different slope degree, the distribution of high and middle vegetation coverage area was assumed as single peak, while the distribution of low vegetation coverage area was decreasing. The high vegetation coverage area was larger than middle and low vegetation coverage area above 25 degree. For different slope direction, the low and middle vegetation coverage area was larger in sunny slope, by contrast, while in the shady slope, the situation was just the opposite.
\end{abstract}

\section{Introduction}

Vegetation is a tie connected with natural elements such as atmosphere, soil and water, and so on. It played an important role in soil and water conservation, atmospheric regulation and ecosystem stability[1]. Moreover, vegetation spatial pattern not only affects ecosystem structure and function, but also reflects soil erosion[2]. Therefore, the vegetation pattern becomes the key to study regional ecosystem structure and soil erosion control[3]. However, traditional vegetation survey method consumes a lot of manpower, material resources, and have poor timeliness, it is difficult to monitor large-scale vegetation distribution. With rapid development of the remote sensing technology, which provides a new method for vegetation monitoring[4]. The normalized difference vegetation index (NDVI) can reflect the degree of vegetation coverage by analysis the vegetation capacity absorbed the effective radiation of light. It has become an important method to study on vegetation pattern[5]. Zhang et al.[6] analyzed the vegetation pattern and variation trend of mid-subtropical forest by NDVI data in Jinggangshan city. Liu et al.[7] evaluated vegetation variability from Landsat images over multiple growing seasons. Eckert et al.[8] analyzed the variable trait of vegetation for detecting land degradation and regeneration in Mongolia using the NDVI time series data. Therefore, in this study, Nanchong city as the research region, based on the MODIS NDVI dataset and terrain factor data derived from digital elevation model (DEM), and analysis the vegetation spatial distribution characteristics by overlaying them using the technology of Geographic Information System. It hoped that this study can provide reference for optimizing land use structure and protecting ecological environment, and preventing soil and water erosion. 


\section{The study area}

The Nanchong city of Sichuan Province is located within $30^{\circ} 35^{\prime} \sim 31^{\circ} 51^{\prime} \mathrm{N}$ and $105^{\circ} 27^{\prime} \sim 106^{\circ} 58^{\prime} \mathrm{E}$. The area is $12479.96 \mathrm{~km}^{2}$ (Fig.1). The region has a humid subtropical climate, with the annual mean temperature of $17^{\circ} \mathrm{C}$, and the annual average rainfall is $1100 \mathrm{~mm}$, which concentrated during the period from July to September. The terrain tilts northeast-southwest, with the elevation of 256-889 m. The soil type is mainly purplish soil and paddy soil, in which the area of purplish soil accounts for $60 \%$ of the total area. The main food are wheat (Triticum aestivum L.), rice (Oryza glaberrima), corn (Zea mays L.) and sweet potato (Ipomoea batatas).

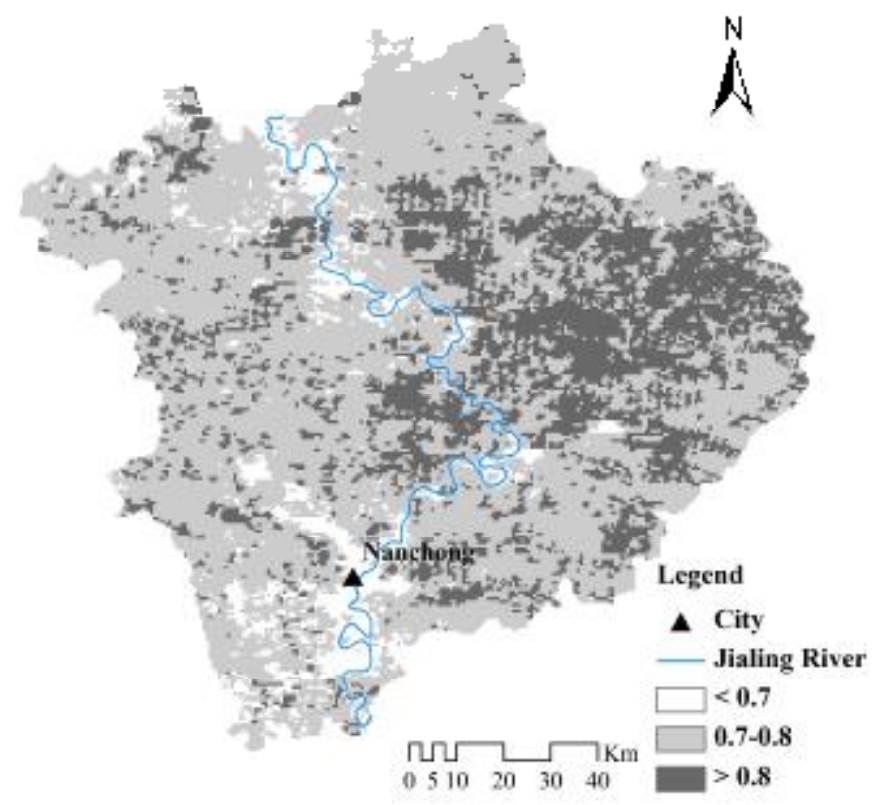

Fig. 1 NDVI spatial distribution in Nanchong city

\section{Materials and methods}

\section{Data sources and processing}

The MODIS NDVI data (resolution $500 \mathrm{~m}$ ) derived from Moderate Resolution Imaging Spectroradiometer (MODIS) in Terra satellite and digital elevation model (DEM) were provided by the International Scientific \& Technical Data Mirror Site, Computer Network Information Center, Chinese Academy of Sciences (http://www.gscloud.cn). The data were a total of 3 phase from July to September in 2015. Using ArcGIS 10.1 to extract the DEM and NDVI of Nanchong. The maximum value composite method was used to obtain NDVI, the purpose was to eliminate the interference such as atmospheric, radiation and cloud, and to ensure the authenticity of the surface vegetation coverage[9]. The method was as follows:

$$
I_{\mathrm{NDVI}}=\max \left(I_{\mathrm{NDVIj}}\right)
$$

Where $I_{\text {NDVI }}$ represents maximum composite value of NDVI; $I_{\text {NDVIj }}$ represents the NDVI value of $j$ th time.

\section{Analysis method}

According to the previous studies[10] and the actual situation of the study area, the NDVI value was divided into 3 grades: the low vegetation coverage (NDVI $\leq 0.70)$, middle vegetation coverage $(0.70<\mathrm{NDVI} \leq 0.80)$ and high vegetation coverage $(\mathrm{NDVI}>0.80)$.

Based on the ArcGIS10.1 platform, the terrain factor maps were obtained from DEM using the spatial analysis tools, and which were classified (Table 1) to generate topographic factors grade maps. 
Finally, the topographic feature distribution of vegetation coverage was analyzed by overlaying the map of each topographic factor and the vegetation coverage.

Table 1 Grade system of topographic factors

\begin{tabular}{ccccc}
\hline \multirow{2}{*}{ topographic factors } & \multicolumn{4}{c}{ Grade } \\
\cline { 2 - 5 } & 1 & 2 & 3 & 4 \\
\hline Altitude $[\mathrm{m}]$ & $<300$ & $300-400$ & $400-500$ & $>500$ \\
Slope-degree $\left.{ }^{\circ}\right]$ & $<10$ & $10-25$ & $25-40$ & $>40$ \\
Slope-direction & Shady slopes & Sunny slpoes & - & - \\
\hline
\end{tabular}

\section{Results and analysis}

\section{Vegetation spatial distribution characteristics}

We can find the spatial distribution characteristics of vegetation coverage in Nanchong through the Fig.1. The low vegetation cover mainly distributed in the Jialing river valley and plain, which accounted for $8.71 \%$ of the total area. However, the middle vegetation coverage mainly distributed in the western region, accounted for $65.85 \%$ of the total area. The high vegetation cover mainly distributed in the eastern regions, accounted for $25.44 \%$ of the total area.

\section{The distribution characteristics of vegetation coverage in altitude}

For altitude, the altitudinal distribution characteristics of vegetation coverage showed in Table 2 . The area percentage in all of the vegetation coverage grade were assumed as single peak at elevation. The maximum area of high, middle and low vegetation coverage were all located in $300-500 \mathrm{~m}$. While the low vegetation coverage area was mainly located below $500 \mathrm{~m}$, high vegetation coverage area was mainly located above $500 \mathrm{~m}$, which indicated that the vegetation was better with the increasing elevation. This is mainly due to the under the $500 \mathrm{~m}$ the terrain was low hills and middle hills region, in which the main land use types were urban land and farm land, and vegetation coverage was weak. But forestland and grassland were main land use types above $500 \mathrm{~m}$.

Table 2 The vegetation coverage area percentage in different topographic factors [\%]

\begin{tabular}{cccccccccc}
\hline Topographic & \multicolumn{4}{c}{ Altitude } & \multicolumn{3}{c}{ Slope degree } & \multicolumn{3}{c}{ Slope direction } \\
\cline { 2 - 10 } factors & Low & Mid & High & Low & Mid & High & Low & Mid & High \\
\hline 1 & 27.20 & 4.53 & 2.14 & 63.87 & 44.91 & 39.13 & 49.20 & 49.70 & 50.21 \\
2 & 57.04 & 50.93 & 47.05 & 32.63 & 46.89 & 48.87 & 50.80 & 50.30 & 49.79 \\
3 & 13.48 & 30.63 & 31.66 & 3.32 & 7.85 & 11.27 & - & - & - \\
4 & 2.29 & 13.92 & 19.15 & 0.18 & 0.34 & 0.73 & - & - & - \\
\hline
\end{tabular}

The distribution characteristics of vegetation coverage in slope degree

For the slope degree, the distribution characteristics of vegetation coverage showed in Table 2. The area percentage of middle and high vegetation coverage was assumed as single peak at slope degree, but that of low vegetation coverage showed a decreasing distribution. The maximum area of high and middle vegetation coverage were located in $10^{\circ} \sim 25^{\circ}$. While the low vegetation coverage area was mainly located below $25^{\circ}$, high vegetation coverage area was mainly located above $25^{\circ}$. The Main reason was that the steep slope land above $25^{\circ}$ was prohibited reclamation doe to the project of Grain for Green implemented. Moreover, with the increasing slope degree, land use types were transformed from construction land and farmland to grassland and forest land, which also resulted in the increase of vegetation coverage.

\section{The distribution characteristics of vegetation coverage in slope direction}

For the slope direction, the distribution characteristics of vegetation coverage showed in Table 2 . In the shady slope the area of vegetation coverage is: high vegetation coverage $>$ middle vegetation coverage >low vegetation coverage, while in the sunny slope that is: low vegetation coverage $>$ middle vegetation coverage $>$ high vegetation coverage. This was caused by the differences water and heat conditions in difference slope direction. Because the better light conditions was, the more 
construction land and cultivated land was, and the poor the vegetation coverage. However, the vegetation evaporation was less, which was conductive to high vegetation coverage growth.

\section{Conclusions}

This study analyzed the spatial distribution characteristics of vegetation coverage under different topographic factors using MODIS NDVI (resolution $500 \mathrm{~m}$ ) date and GIS technology in Nanchong, Sichuan province, China. The main conclusions were as follows:

(1) The vegetation coverage was dominated by middle and high coverage in Nanchong, average NDVI value was 0.76 , indicating good ecological environment. The high vegetation coverage distributed in the eastern region, while the middle vegetation coverage distributed in the western region. Low vegetation coverage distributed in the Jialing River valley, especially in urban area.

(2)At the different elevation and slope degree, NDVI mean is rising. In which the largest vegetation coverage area of high, medium and low appeared at 300-500 $\mathrm{m}$ in different altitude. And in different slope degree, the distribution of high and middle vegetation coverage area was assumed as single peak, while the distribution of low vegetation coverage area was decreasing. The high vegetation coverage area was larger than middle and low vegetation coverage area above 25 degree. For different slope direction, the low and middle vegetation coverage area was larger in sunny slope, by contrast, while in the shady slope, the situation was just the opposite.

\section{Acknowledgements}

This work was financially supported by the Special Foundation of China West Normal University (13D003).

\section{References}

[1] Jiang W, Yuan L, Wang W, Cao R, Zhang Y, Shen W. Spatio-temporal analysis of vegetation variation in the Yellow River Basin. Ecological Indicators. 2015;51:117-126.

[2] Kileshye Onema J-M, Taigbenu A. NDVI-rainfall relationship in the Semliki watershed of the equatorial Nile. Physics and Chemistry of the Earth, Parts A/B/C. 2009;34:711-721.

[3] Setiawan Y, Yoshino K, Prasetyo LB. Characterizing the dynamics change of vegetation cover on tropical forestlands using 250m multi-temporal MODIS EVI. International Journal of Applied Earth Observation and Geoinformation. 2014;26:132-144.

[4] Anyamba A, Tucker CJ. Analysis of Sahelian vegetation dynamics using NOAA-AVHRR NDVI data from 1981-2003. Journal of Arid Environments. 2005;63:596-614.

[5] Costantini ML, Zaccarelli N, Mandrone S, Rossi D, Calizza E, Rossi L. NDVI spatial pattern and the potential fragility of mixed forested areas in volcanic lake watersheds. Forest Ecology and Management. 2012;285:133-141.

[6] Zhang J, Zhang L, Xu C, Liu W, Qi Y, Wo X. Vegetation variation of mid-subtropical forest based on MODIS NDVI data - A case study of Jinggangshan City, Jiangxi Province. Acta Ecologica Sinica. 2014;34:7-12.

[7] Liu J, Pattey E, Jégo G. Assessment of vegetation indices for regional crop green LAI estimation from Landsat images over multiple growing seasons. Remote Sensing of Environment. 2012;123:347-358.

[8] Eckert S, Hüsler F, Liniger H, Hodel E. Trend analysis of MODIS NDVI time series for detecting land degradation and regeneration in Mongolia. Journal of Arid Environments. 2015;113:16-28.

[9] Du J, Shu J, Yin J, Yuan X, Jiaerheng A, Xiong S, et al. Analysis on spatio-temporal trends and drivers in vegetation growth during recent decades in Xinjiang, China. International Journal of 
Applied Earth Observation and Geoinformation. 2015;38:216-228.

[10] Fu B, Burgher I. Riparian vegetation NDVI dynamics and its relationship with climate, surface water and groundwater. Journal of Arid Environments. 2015;113:59-68. 